\title{
Clinical Characteristics and Laboratory Abnormalities of Hospitalized and Critically III Children with Coronavirus Disease 2019: A Retrospective Study from Saudi Arabia
}

\author{
Ali Alsuheel Asseri ${ }^{1}$ \\ Ibrahim Alzaydani ${ }^{2}$ \\ Ahmed Al-Jarie ${ }^{2}$ \\ Ahmed Albishri ${ }^{3}$ \\ Abdullah Alsabaani ${ }^{4}$ \\ Mohammed Khamash \\ Almaghrabi (D) $^{5}$ \\ Abdelwahid Saeed Ali $\mathbb{1 D}^{5}$ \\ 'Department of Child Health, College of \\ Medicine, King Khalid University, Abha, \\ Saudi Arabia; ${ }^{2}$ Department of Pediatrics, \\ Abha Maternity and Children Hospital, \\ Abha, Saudi Arabia; ${ }^{3}$ Department of \\ Pediatrics, Khamis Military Hospital, \\ Ministry of Defense, Khamis Mushait, \\ Saudi Arabia; ${ }^{4}$ Department of Family and \\ Community Medicine, College of \\ Medicine, King Khalid University, Abha, \\ Saudi Arabia; ${ }^{5}$ Department of \\ Microbiology and Clinical Parasitology, \\ College of Medicine, King Khalid \\ University, Abha, Saudi Arabia
}

Background: COVID-19 was reported in several studies characterized by milder clinical course, benign disease, and peculiar epidemiologic patterns among pediatric patients compared to adults' disease. However, other studies indicated that critical cases also exist and are associated with preexisting cardiopulmonary comorbidities and concurrent multisystem inflammatory syndrome in children.

Methods: The study period was six months, May-October 2020. Data on demographics, clinical manifestations, laboratory abnormalities were extracted from the patients' hospital records. During the study period, 644 pediatric patients attended the hospital. They were all screened for SARS-CoV-2 using RT-PCR. Only the confirmed positive patients were included in the subsequent study analysis. They were hospitalized either in the general pediatric wards (GPW) or pediatric intensive care unit (PICU).

Results: Out of the total patients screened, 79 (12.3\%) children were confirmed to have COVID-19 infection. All the confirmed COVID-19 patients were either admitted to the general pediatric wards $(58 ; 73.4 \%)$ or PICU $(21 ; 26.6 \%)$. The admission diagnoses for these children were acute gastroenteritis $(22.85 \%)$, acute pneumonia $(19 \%)$, clinical sepsis $(17.7 \%)$, and multisystem inflammatory syndrome in children (10.1\%). A significantly higher percentage of the PICU admitted patients showed shortness of breath (SOB) $(P=0.016)$. Respiratory insufficiencies, prematurity, and congenital heart diseases are the most reported comorbid conditions among the admitted children. The oxygen saturation was significantly lower among PICU patients than those in GPW $(P=0.001)$. The total hospital stays differ significantly between the two groups, which were ten days for the PICU group compared to 4.5 days for the GPW group with a statistical significance noted $(\mathrm{P}=0.001)$.

Conclusion: Despite the observable variations in the clinical and laboratory findings among the hospitalized pediatric COVID-19 patients, no serious consequences among all patients were observed. The history of SOB and the initial oxygen saturation level were significantly associated with PICU admissions.

Keywords: children, COVID-19, SARS-CoV-2, PICU, pediatric general ward

\section{Introduction}

Department of Child Health, College of Medicine, King Khalid University, Abha, Saudi Arabia

Tel +9665001860I3

Email alsoheelI I@kku.edu.sa
A novel coronavirus named severe acute respiratory syndrome coronavirus 2 (SARSCoV-2) first reported in China in December 2019, causing severe respiratory infection in humans known COVID-19. ${ }^{1-6}$ The infection was first seen as responsible for the 
massive and fatal epidemic in China, then rapidly spread worldwide, resulting in a global pandemic with high morbidity and mortality rates. ${ }^{7-10}$ COVID-19 was reported to affect all age groups, including neonates, infants, children, adults, and the elderly. ${ }^{11}$ Although earlier reports of COVID-19 among children indicated that the disease is characterized by a milder course and a high prevalence of asymptomatic cases than adult COVID-19, later studies reported severe and critical pediatric COVID-19 infections. ${ }^{11-18}$ Several epidemiological studies have also identified the risk factors of severe COVID-19 in children, such as pre-existing conditions like cardiac, respiratory, and immunodeficiency disorders and the concurrent hyperinflammatory syndrome named multisystem inflammatory syndrome-children (MISC). ${ }^{14,15,17}$ In Saudi Arabia, many reports for COVID-19 described the clinical onset, symptomatology, epidemiology, pathology, medical interventions, and effectiveness of the disease's preventive measures in total and pediatric patients on a particular basis. ${ }^{19-22}$ To our knowledge, this is the first report on the pediatric COVID-19 among hospitalized and critically ill patients in Saudi Arabia. In this paper, we report and discuss the clinical outputs and the consequences of COVID-19 among the severely infected and pediatric intensive care unit (PICU) hospitalized children in the study area in Saudi Arabia. We also compare the disease course and clinical phenotypes among these patients with those admitted to the general pediatric ward (GPW) in the Asir region, Southwest of Saudi Arabia.

\section{Methods}

\section{Study Population}

This is a retrospective study that included children hospitalized between May 2020 and October 2020 at Abha Maternity and Children Hospital (AMCH) and Khamis Military Hospital, Southwest of Saudi Arabia. These two hospitals are considered a tertiary care and teaching hospitals at the southwestern region of the Kingdom of Saudi Arabia. The estimated children in the catchment area of the hospitals are 200,000. All children included in this study aged between 8 days to 12 years old. They were laboratory-confirmed COVID-19 infected children using the reverse-transcription polymerase-chain-reaction (RTPCR) testing of the nasopharyngeal swab specimens. The screening for SARS-CoV-2 followed the national guidelines set by the Ministry of Health (MOH) ${ }^{23}$ Saudi Arabia, and the Saudi Center of Disease Control and Prevention for children. ${ }^{24}$

\section{Study Design and Setting}

Based on the initial emergency room physician assessment and the $\mathrm{MOH}$ pediatric COVID-19 admission criteria, patients were admitted to the GPW or PICU. The criteria for GPW admissions include Infants aged $<1$ year, clinical or radiographic evidence of pneumonia, oxygen saturation $<92 \%$ on room air, pre-existing chronic condition (moderate to severe asthma, cardiovascular disease, chronic kidney disease, chronic liver disease, neuromuscular disease, diabetes Mellitus, and metabolic disorders), immunocompromised patient, clinical features that are similar to those of toxic shock syndrome and atypical Kawasaki disease, gastroenteritis picture with dehydration and/or poor oral intake, persistent high fever for 3-5 days, and disease course longer than 1 week, and no improvements in symptoms or signs or progressive exacerbation, while the criteria for PICU admission include hypoxic patients on more than 5 LPM oxygen or noninvasive/invasive ventilations, patients with multisystem inflammatory syndrome, severely dehydrated patients with acute kidney injury (urea and creatinine more than the normal for age), prolonged seizures for more than 5 minutes with no response to initial therapies, diabetic ketoacidosis, and patients with severe electrolyte disturbances. After confirmation of COVID-19 infection, the treating team started the patient's management according to the Saudi $\mathrm{MOH}$ COVID-19 protocols of pediatric patients. ${ }^{25}$ Special focus and attention were made on children characterized by MIS-C conditions. The definition of MIS-C included the following criteria (as per the US CDC definition): an age of less than 21 years, fever $>38.0^{\circ} \mathrm{C}$ lasting at least 24 hours, laboratory evidence of inflammation, multisystem organ involvement (ie, involving at least two systems), and laboratory-confirmed SARS-CoV-2 infection (positive SARS CoV-2 with realtime reverse-transcriptase polymerase chain reaction (RTPCR)). ${ }^{26}$ In this study, all patients with the diagnosis of MIS-C and negative concurrent SARS-CoV-2 infection were excluded. Sepsis, severe sepsis, and septic shock were defined as per the pediatric surviving sepsis guideline. ${ }^{27} \mathrm{King}$ Khalid University Institutional Review Board (KKU-IRB) reviewed and approved this retrospective study.

\section{Data Collection}

Demographic data, initial symptoms and signs, laboratory results, treatment interventions, and outcomes were extracted from the hospital medical records. The presence of comorbidities like (asthma, congenital heart disease, 
genetic syndromes, epilepsy, and chronic lung disease) were also documented.

\section{Statistical Analysis}

After the data were collected, coded, and entered into statistical software (IBM SPSS version 22, SPSS, Inc. Chicago, IL). All statistical analyses were made using the two-tailed tests. A P-value of less than 0.05 was considered statistically significant. Descriptive analysis based on the frequency and percent distribution was done for all variables, including demographic data, admission site, symptoms, medications, and comorbidities. Means with standard deviations were calculated for the normally distributed quantitative variables, while median with interquartile range (IQR) was calculated for skewed numerical variables. Cross tabulation was used to test for the distribution of COVID-19 infected children according to their bio-clinical data and admission site (GPW/PICU). Pearson's Chi-square test was also used to test for relations significance. Exact tests were used to assess significant association due to small frequencies. For the normally distributed variables, an independent $t$-test was used for comparing means, while the Mann-Whitney test was used for the skewed variables. Odds ratios were calculated for the statistically significant variables by using bivariate logistic regression.

\section{Ethical Approval}

The study was approved by the Institutional Research Ethics (IRE) board, the College of Medicine's ethical committee, King Khalid University (KKU). It was carried out according to the Declaration of Helsinki. Patients' informed consents were not needed since this study was a retrospective observational study without any interventions.

\section{Results}

\section{Preadmission Data of the Pediatric Patients}

From May to October 2020, a total of 644 children were screened for SARS-CoV-2 infection. Seventy-nine $(12.3 \%)$ of these pediatric patients were confirmed positive with the RT-PCR test. Twenty-one (26.6\%) of these patients required PICU admission (Figure 1). Most of the children who required PICU admission were more than five years old, while most GPW admissions were for children between 1 and 5 years old (Figure 2).

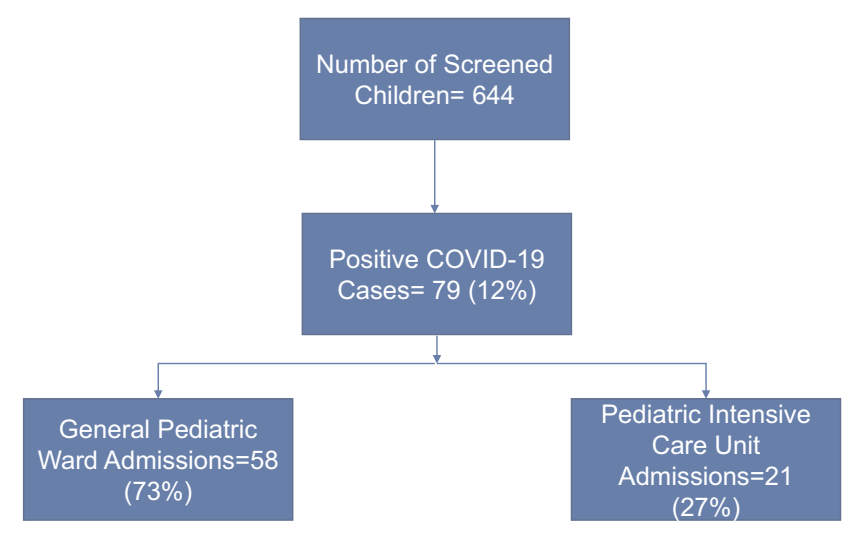

Figure I The flowchart showing the number of confirmed COVID-19 cases and the admission settings (general pediatric ward and pediatric intensive care unit).

The highest admission rate was reported during the summer months (June, July, and August), while lower rates were observed during September and October (Figure 3). The categories of final diagnoses for patients who were tested positive for COVID-19 (79 patients) were as follows, acute gastroenteritis $18(22.85 \%)$, acute pneumonia 15 (19\%), clinical sepsis 14 (17.72\%) (Figure 4).

\section{Baseline Demographics and Clinical Characteristics of Pediatric COVID-19}

Table 1 summarized the detailed baseline demographic and clinical characteristics of the enrolled patients. The hospitalized children ranged between newborns to 144 months with a median age of 24 months. A number of 44 patients $(55.7 \%)$ were males. A history of contact to COVID-19 cases was reported by 67 (84.8\%) children. There were no significant variations observed among those admitted to PICU or GPW as per the age, gender, and history of contacts to COVID-19 patients. For the distribution of children's ages by their place of admission, the median age of those who were admitted to PICU was 60 months compared to 14 months for children who were admitted to the GPW with no statistical significance detected $(\mathrm{P}=0.211)$. An exact $71.4 \%$ of those who were admitted to PICU were males compared to $50 \%$ of GPW admitted children ( $\mathrm{P}=0.074)$. Contact to infected COVID19 cases were reported among 88\% of GPW admitted children compared to $76.2 \%$ of PICU admitted children.

The most reported symptoms among PICU admitted children were fever $(100 \%)$, cough $(71.3 \%)$, and shortness of breath (SOB) (67\%). SOB proportions were significantly higher among PICU children than GPW children ( 67 vs $36 \%$; $\mathrm{P}=0.016$ ). In addition, $62 \%$ of the children 


\section{5}

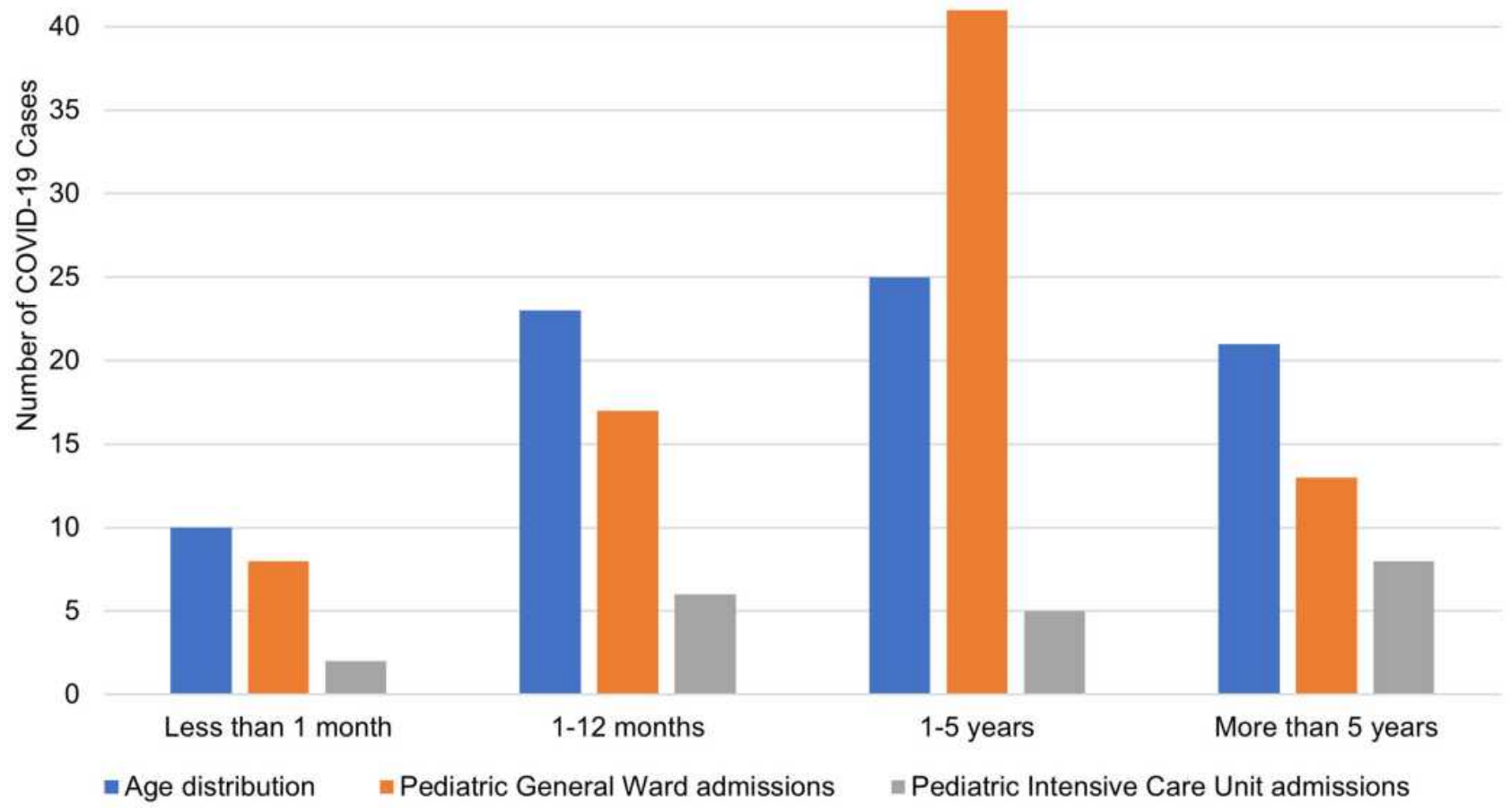

Figure 2 Age distribution of hospitalized COVID-19 cases, general pediatric ward, and pediatric intensive care unit admissions.

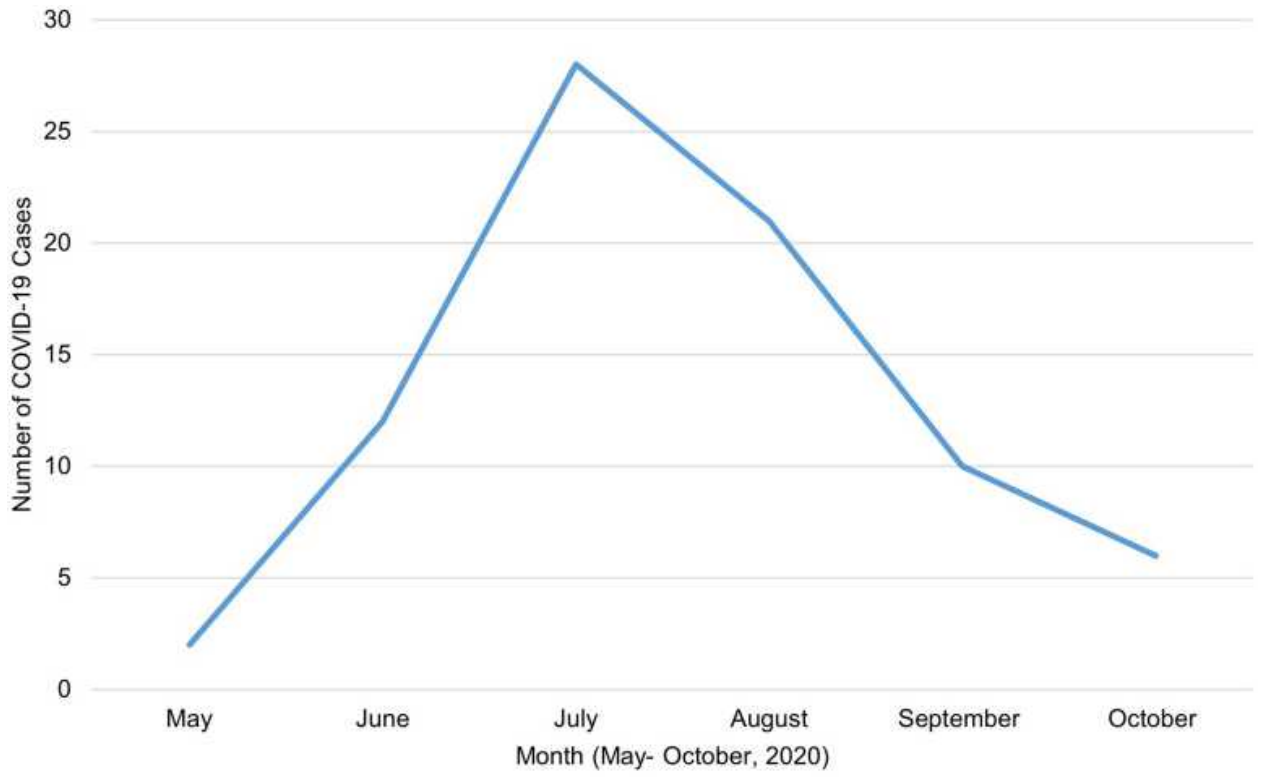

Figure 3 Distribution of COVID-19 cases by month (May-October 2020).

who were admitted to the GPW were previously healthy compared to $48 \%$ of PICU admitted children. The mostreported comorbidities among the PICU in comparison to GPW admitted children were respiratory insufficiency ( $19 \%$ vs $22 \%$ ), prematurity ( $14 \%$ vs $19 \%$ ), and CHD
(14\% vs $9 \%$ ). The pre-existing hematological disorders (sickle cell disease and leukemias) were reported by only three children who were admitted to the GPW. The median temperature among PICU children was 37.9 compared to 38.0 among the GPW admitted children with no statistical 


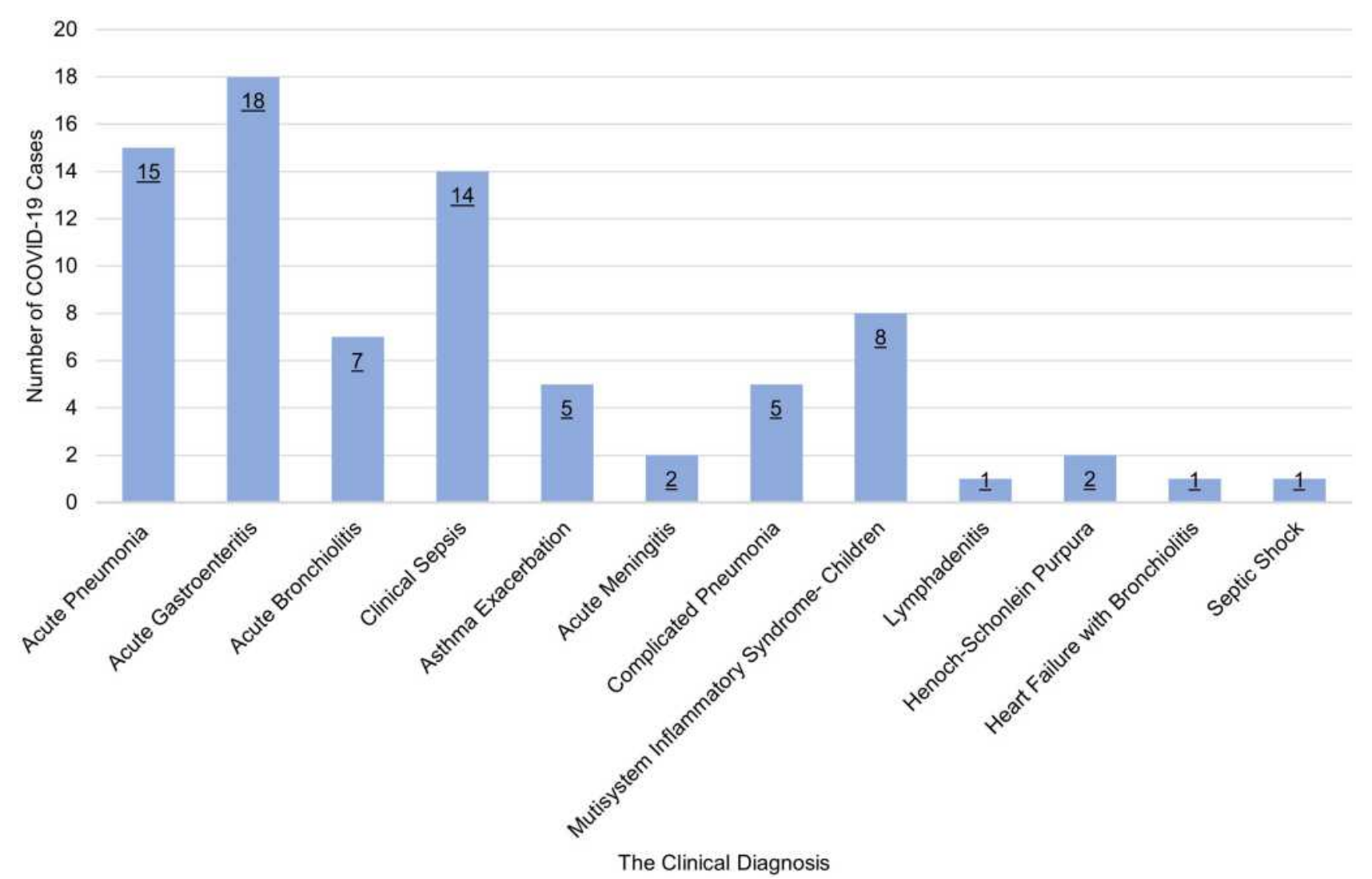

Figure 4 Clinical diagnosis of confirmed COVID-19 cases.

significance noted $(\mathrm{P}=0.726)$. The oxygen saturation was detected significantly lower among PICU admitted children in contrast to the GPW patients (85\% vs $95 \%)$ (P $<0.001)$. History of SOB and the initial emergency room (ER) oxygen saturation were associated with PICU admission; the odds ratios and $\mathrm{p}$-values were 3.5; P-value 0.022 and $-3.2 ;$ P-value $<0.001$, respectively.

\section{Laboratory Findings of COVID-19 Pediatric Patients}

Table 2 illustrates the laboratory findings among GPW and PICU admitted children. Absolute neutrophil counts (ANC) were higher among PICU admitted children than GPW admitted children (8791 vs 5364, respectively; P= 0.021 ). The same trend of the variations between the two groups of children was also observed for the ESR ( $38 \pm 8$ vs $24 \pm-4$, respectively, $\mathrm{P}=0.050)$ and ferritin levels $(710 \pm 240$ vs $171 \pm 28$, respectively, $\mathrm{P}<0.001)$. Lower albumin levels among the children admitted to the PICU than among the GPW children were noted (2.8 vs 3.6 , respectively, $\mathrm{P}$ $<0.001)$. There was a trend of high procalcitonin, D-dimer, and CRP levels among PICU admitted patients compared to those admitted to the GPW with no statistical significance observed.

\section{Therapies and Outcome Measures of the COVID-19 Patients}

The therapies and outcome measures among ward and PICU admitted groups of children are demonstrated in Table 3. As for pain and fever therapies, the most prescribed treatment among PICU patients compared to GPW patients was paracetamol ( $100 \%$ vs $82 \%$; $\mathrm{P}=0.038)$. Hydroxychloroquine was also administered to both PICU and GPW admitted children (75\% vs $71 \%$, respectively; $\mathrm{P}=0.498$ ). Systemic steroids (methylprednisolone and oral prednisolone) and Favipiravin were often administered to PICU more than GPW patients ( $62 \%$ vs $25 \% ; \mathrm{P}=0.003)$ and ( $29 \%$ vs $2 \% ; \mathrm{P}<0.001)$, respectively, with statistical variations observed. Considering other medications, the most reported for PICU group versus GPW group were Nebulizer bronchodilators $(71 \%$ vs $28 \%$; $\mathrm{P}=0.001$ ), followed by intravenous immunoglobulin (IVIG) ( $33 \%$ vs $0.0 \%, \mathrm{P}<0.001$ ). Regarding broad-spectrum antibiotic therapies, cefuroxime was the most prescribed antibiotic for GPW patients compared to PICU patients (75\% vs $33 \%, \mathrm{P}=$ 
Table I Baseline Demographic and Clinical Characteristics of the COVID-I 9 Pediatric Patients

\begin{tabular}{|c|c|c|c|c|}
\hline Variables & Total $\mathbf{n = 7 9}$ & Pediatric Ward n=58 (73.4\%) & PICU n=2I (26.6\%) & $P$ value \\
\hline Age in months, median (IQR) & $24(3-72)$ & $14(2-60)$ & $60(8-84)$ & 0.210 \\
\hline Gender, male, No. (\%) & $44(56 \%)$ & $29(50 \%)$ & I5 (7I.4\%) & 0.074 \\
\hline History of contact with COVID-19, No. (\%) & $67(84.8 \%)$ & $51(88 \%)$ & $16(76.2 \%)$ & 0.175 \\
\hline $\begin{array}{l}\text { Symptoms on presentation, No. (\%) } \\
\text { - Fever } \\
\text { - Cough } \\
\text { - Shortness of breath (SOB) } \\
\text { - Sore throat } \\
\text { - Runny nose } \\
\text { - Vomiting } \\
\text { - Diarrhea } \\
\text { - Convulsion }\end{array}$ & $\begin{array}{c}76(96 \%) \\
44(55.7 \%) \\
35(44.3 \%) \\
20(25 \%) \\
24(30.4 \%) \\
27(34.2 \%) \\
23(29 \%) \\
5(6.3 \%)\end{array}$ & $\begin{array}{c}55(94.8 \%) \\
29(50 \%) \\
21(36 \%) \\
13(22 \%) \\
15(26 \%) \\
20(35 \%) \\
18(31 \%) \\
3(5.2 \%)\end{array}$ & $\begin{array}{c}21 \text { ( } 100 \%) \\
15(7 \mid .3 \%) \\
14(67 \%) \\
7(33 \%) \\
9(43 \%) \\
7(33 \%) \\
5(24 \%) \\
2(10 \%)\end{array}$ & $\begin{array}{l}0.288 \\
0.074 \\
0.016^{*} \\
0.241 \\
0.121 \\
0.574 \\
0.372 \\
0.401\end{array}$ \\
\hline $\begin{array}{l}\text { Comorbid conditions, No. (\%) } \\
\text { - Previously Healthy } \\
\text { - Asthma } \\
\text { - Respiratory insufficiency } \\
\text { - Rheumatological Disorders } \\
\text { - Hematological Disorders } \\
\text { - Genetic Disorder } \\
\text { - Epilepsy } \\
\text { - CHD } \\
\text { - Home oxygen use } \\
\text { - Prematurity }\end{array}$ & $\begin{array}{c}46(58 \%) \\
10(13 \%) \\
17(22 \%) \\
2(3 \%) \\
3(3.8 \%) \\
5(6 \%) \\
5(6.3 \%) \\
8(10 \%) \\
5(6.33 \%) \\
14(18 \%)\end{array}$ & $\begin{array}{c}36(62 \%) \\
8(14 \%) \\
13(22 \%) \\
2(3.5 \%) \\
3(5.2) \\
3(5.2 \%) \\
4(7 \%) \\
5(9 \%) \\
3(5.2 \%) \\
11(19 \%)\end{array}$ & $\begin{array}{c}10(48 \%) \\
2(10 \%) \\
4(19 \%) \\
0(00 \%) \\
0(00 \%) \\
2(10 \%) \\
1(5 \%) \\
3(14 \%) \\
2(10 \%) \\
3(14 \%)\end{array}$ & $\begin{array}{c}0.186 \\
0.47 I \\
0.507 \\
0.389 \\
0.039 * \\
0.401 \\
0.599 \\
0.359 \\
0.401 \\
0.455\end{array}$ \\
\hline $\begin{array}{l}\text { Initial vital signs, median (IQR) } \\
\text { - Temperature } \\
\text { - Oxygen saturation }\end{array}$ & $\begin{array}{c}37.9 \pm 0.1 \\
94 \%(88-96)\end{array}$ & $\begin{array}{c}38 \pm 0.1 \\
95 \%(91-96)\end{array}$ & $\begin{array}{c}37.9 \pm 0.1 \\
85 \%(80-93)\end{array}$ & $\begin{array}{c}0.726 \\
<0.00 I^{*}\end{array}$ \\
\hline
\end{tabular}

Note: $* \mathrm{P}<0.05$ (significant).

$0.004)$, while ceftriaxone was often prescribed to PICU than to GPW patients $(62 \%$ vs $21 \% ; \mathrm{P}=0.001)$. The median time for the total hospital stay was ten days for the PICU group compared to 4.5 days for the GPW group with statistical significance $(\mathrm{P}<0.001)$. One patient $(1.3 \%)$ died due to severe septic shock and multiorgan failures despite receiving all supportive therapies that included mechanical ventilation, vasopressors, IVIG, methylprednisolone, and antibiotics.

\section{Discussion}

Since the beginning of the COVID-19 pandemic in December of 2019, several studies showed that children could be infected but get milder disease with fewer longterm morbidities and mortalities compared to the adult population. ${ }^{11,13,17,18,28-30}$ Over a period of six months (May to October 2020), at two pediatric departments in the Southwest province of Saudi Arabia, 79 out of 644 screened pediatric patients $(12.3 \%)$ had tested positive for
COVID-19. Similar previous studies have reported different percentages of positive COVID-19 cases among children populations in the particular community ranging between $10 \%$ and $30 \% .^{12,17,18}$ Again, this substantiates the low infectivity rates and benign disease among pediatric settings in many parts of the world. Explanations for these low infectivity rates of the disease among children were intensively reviewed and reported from virological, biochemical, clinical, epidemiological, and behavioral perspectives. $^{11}$ More than $25 \%$ of our cohort patients needed critical care admissions mainly due to respiratory failure with pre-existing cardiac or respiratory conditions and MIS-C. Our data concur with the previous studies, which reported the ICU admission levels in children due to COVID-19 range between $10 \%$ and $28 \%{ }^{1,2,8}$ The peak of our COVID-19 cases was noted in the mid of July, which mirrors the international and national peak of COVID-19 cases. $^{29,31}$ 
Table 2 Initial Laboratory Findings of the COVID-19 Pediatric Patients

\begin{tabular}{|c|c|c|c|c|}
\hline Variables & Total $n=79$ & Pediatric Ward $n=58$ (73.4\%) & $P I C U n=2 I n=2 I \quad(26.6 \%)$ & $P$ value \\
\hline WBC (Ref: $\left.4.3-11.0 \times 10^{3} / \mu \mathrm{L}\right)$, mean \pm SD & $11.5 \pm 0.95$ & $10.4 \pm 0.8$ & $14.3 \pm 2.6$ & 0.063 \\
\hline ANC (Ref: $1500-8500$ cells/microL), mean \pm SD & $6323 \pm 774$ & $5364 \pm 662$ & $8791 \pm 2124$ & $0.021 *$ \\
\hline ALC (Ref: $970-3960 / \mu \mathrm{L})$, mean \pm SD & $3906 \pm 345$ & $3808 \pm 315$ & $4157 \pm 945$ & 0.653 \\
\hline Hemoglobin (Ref: II.5- $15.5 \mathrm{~g} / \mathrm{dL}$ ), mean \pm SD & $11.7 \pm 2$ & $11.5 \pm 2$ & $12.4 \pm 2$ & 0.201 \\
\hline C-reactive protein (Ref: $0.0-0.9 \mathrm{mg} / \mathrm{dL}$ ), mean \pm SD & $26 \pm 11.2$ & $15 \pm 9.4$ & $47 \pm 27.3$ & 0.177 \\
\hline ESR (Ref: $0.0-15 \mathrm{~mm} / \mathrm{hr}$ ), mean \pm SD & $28 \pm 3.7$ & $24 \pm 4$ & $38 \pm 8$ & $0.050 *$ \\
\hline Ferritin (Ref: $13.7-78.8 \mathrm{ng} / \mathrm{mL}$ ), mean $\pm \mathrm{SD}$ & $321 \pm 76$ & $|7| \pm 28$ & $710 \pm 240$ & $<0.001^{*}$ \\
\hline D-dimer (Ref: $0.0-500 \mathrm{pg} / \mathrm{mL}$ ), mean $\pm \mathrm{SD}$ & $830 \pm 191$ & $603 \pm 117$ & $1316 \pm 535$ & 0.082 \\
\hline Procalcitonin (Ref: $0.0-0.1 \mathrm{ng} / \mathrm{mL}$ ), median (IQR) & $0.2(0.08-0.45)$ & $0.12(0.04-0.42)$ & $0.36(0.20-0.47)$ & 0.076 \\
\hline ALT (Ref: 10-35 U/Lb), median (IQR) & $20(14-25)$ & $19.5(14-25)$ & $22(17-28)$ & 0.283 \\
\hline AST (Ref: 10-34 U/L), median (IQR) & $23(19-33)$ & $23.5(19-32)$ & $22(17-48)$ & 0.745 \\
\hline Albumin (g/dL) Ref: $3.7-5.6 \mathrm{~g} / \mathrm{dL}$, median (IQR) & $3.45(2.85-3.8)$ & $3.6(3.3-4.1)$ & $2.8(2.4-3.2)$ & $<0.00 I^{*}$ \\
\hline LDH (Ref 420-750 U/L), median (IQR) & $305(213-430)$ & $292(2|3-4| 3)$ & $340(204-534)$ & 0.602 \\
\hline
\end{tabular}

Note: $* \mathrm{P}<0.05$ (significant).

Abbreviations: ANC, absolute neutrophils count; ALC, absolute lymphocytes count; ESR, erythrocytes sedimentation rate; ALT, alanine aminotransferase; AST, aspartate aminotransferase; LDH, lactate dehydrogenase.

Table 3 Therapies and Outcome Measures of the COVID-19 Patients

\begin{tabular}{|c|c|c|c|c|}
\hline Variables & Total $n=79$ & Pediatric Ward $n=58$ (73.4\%) & PICU $n=2 I(26.6 \%)$ & $P$ value \\
\hline \multicolumn{5}{|l|}{ Pain and fever management, No (\%) } \\
\hline Paracetamol & $58 / 66(88 \%)$ & $37 / 45(82 \%)$ & $21(100 \%)$ & $0.038 *$ \\
\hline Ibuprofen & $34 / 54(63 \%)$ & $25 / 37(68 \%)$ & $9 / 17(53 \%)$ & 0.231 \\
\hline Hydroxychloroquine & $47 / 65$ (72\%) & $32 / 45(71 \%)$ & $15 / 20(75 \%)$ & 0.498 \\
\hline Oseltamivir & $3 / 79(4 \%)$ & $3 / 58(5.2 \%)$ & $0(100)$ & 0.390 \\
\hline Systemic steroid & $27 / 78(35 \%)$ & $14 / 57(25 \%)$ & $|3 / 2|(62 \%)$ & $0.003 *$ \\
\hline Favipiravin & $7 / 79(9 \%)$ & $\mathrm{I} / 58(2 \%)$ & $6 / 21(29 \%)$ & $<0.00 I^{*}$ \\
\hline \multicolumn{5}{|l|}{ Broad-spectrum antibiotics, No (\%) } \\
\hline Ceftriaxone & $25 / 78(32 \%)$ & $12 / 57(2 \mid \%)$ & $|3 / 2|(62 \%)$ & $0.001 *$ \\
\hline Azithromycin & $36 / 53(68 \%)$ & $26 / 36(72 \%)$ & $10 / 17$ (59\%) & 0.252 \\
\hline Cefuroxime & $33 / 54(61 \%)$ & $27 / 36(75 \%)$ & $6 / 18(33 \%)$ & $0.004 *$ \\
\hline Ampicillin and Gentamycin & $10 / 79(13 \%)$ & $8 / 58(14 \%)$ & $2 / 21(10 \%)$ & 0.471 \\
\hline Tazocin & I2/79 (I5\%) & $5 / 58(9 \%)$ & $7 / 21$ (33\%) & $0.012 *$ \\
\hline \multicolumn{5}{|l|}{ Other therapies } \\
\hline Intravenous Immunoglobulin & $7 / 79(9 \%)$ & $0(0.00 \%)$ & $7 / 21(33 \%)$ & $<0.001 *$ \\
\hline Nebulizer bronchodilators & $31 / 79(39 \%)$ & $16 / 58(28 \%)$ & $|5 / 2|(7 \mid \%)$ & $0.001 *$ \\
\hline \multicolumn{5}{|l|}{ Outcome measures, No (\%) } \\
\hline Total hospital stays, median (IQR) & $6(3-10)$ & $4.5(3-7)$ & $10(7.5-12)$ & $<0.001 *$ \\
\hline Discharged, No (\%) & 78/79 (98.7\%) & $58 / 58(100 \%)$ & $20 / 21$ (95.2\%) & 0.610 \\
\hline
\end{tabular}

Note: ${ }^{*} \mathrm{P}<0.05$ (significant).

In agreement with other similar epidemiological studies, ${ }^{3,8,20,21}$ we observed that more boys than girls (56\% vs 44\%) affected with COVID-19, and more boys needed critical care admissions ( $71.4 \%$ vs $28.6 \%)$ than girls. Infants less than one month represent $13 \%$ while $29 \%$ were less than a year; most pediatric ward admissions 
were $1-5$ years of age (52\%). Although the difference between the males' and females' patients in this study is not statistically significant as described in other studies, ${ }^{16}$ however still, logical reasoning for this difference lacks in the literature. Moreover, critical care admissions were mainly observed among male patients with more than five years and had MIS-C diagnoses which concur with other published studies. ${ }^{6,14,31,32}$ Infants below three months of age were observed to have a milder disease with a very low prevalence of PICU admission, which agrees with other studies. ${ }^{33}$

Respiratory symptoms and diarrhea are the most common presenting complaints; shortness of breath occurs in more than two-thirds of critically admitted patients. Diarrhea was reported in around $30 \%$ of our patients, which is in line with other publications. ${ }^{33,34}$ In addition, the initial oxygen saturation at the ER in those who required PICU admissions was $10 \%$ lower compared with ward admissions. Taken all together, SOB, initial oxygen saturation is associated with the PICU admission and the need for further medical interventions.

There were eight cases of MIS-C in our study. These cases had been picked out as the clinicians endowed with high awareness levels about this condition in the hospital settings compared to the previous reports in the pandemic's early course. ${ }^{12,13,18,28}$ The reported MIS-C cases were associated with older age, multisystem involvement, high inflammatory markers, and admission to critical care. In this cohort, there was one death case $(1.3 \%)$ of fiveyear-old males diagnosed with MIS-C and succumbed due to severe septic shock and multiorgan failure despite receiving systemic steroid, antiviral, and mechanical ventilation. On September 15, 2020, the United States Centers for Disease Control and Prevention (CDC) reported the death to be less than $1 \%$ in the pediatric age group, specifically age less than 21 years. ${ }^{15}$

Critical care patients have higher white cell counts and inflammatory markers. Excluding patients with MIS-C, inflammatory markers are still higher in PICU admitted patients but not statistically significant (data are not shown). In addition, serum albumin was lower in the critical care patients compared to GPW patients, which could be explained by a high prevalence of sepsis and MIS-C among PICU patients.

Given the lack of randomized clinical trial-based therapies, there were varieties of interventions used in our cohort. However, the Saudi MOH protocols were the mainframe of therapy guidance. ${ }^{24}$ Paracetamol and NSAID were the most used medicines for fever therapy.
Broad-spectrum antibiotics were used initially for almost all patients; cefuroxime and azithromycin were used for milder cases, while ceftriaxone, tazocin, and ampicillin and gentamycin were used for moderate and severe. The antibiotics were later adjusted after getting the initial culture results (blood, urine, stool, and cerebrospinal fluids), either discontinuing or adjusting the antibiotic coverage based on the culture results. The higher prevalence of antibiotic uses in our study compared to other community-based studies ${ }^{13,27,32}$ could be explained by the nature of our hospital-based data and the high prevalence of preexisting medical conditions. In addition, our cohort included severe and critical COVID-19 patients who were initially sick and needed hospitalizations.

More than $70 \%$ of our patients received hydroxychloroquine (HCQ) with a good safety profile except for one patient who developed mild neutropenia two days after starting HCQ, which returned to normal a week after stopping the HCQ. HCQ had been widely used at the beginning of the pandemic until the US FDA recommended against its use. ${ }^{14,35-37}$ Given the high prevalence of comorbid conditions and MIS-C cases in our cohort, the hospital stays ranged between 3 and 10 days which is slightly higher than the reported length of stay in other similar studies. ${ }^{6}$ A subgroup of patients in our study developed severe disease requiring PICU care characterized by high inflammatory markers (ESR, ferritin, and absolute neutrophil counts) and neutrophil counts compared to general ward admissions, which agree with other studies. ${ }^{14,15}$

Our study has several limitations: First, our data's retrospective and observational nature increase the chance of bias and limit the external generalization. Second, the high prevalence of comorbid conditions and MIS-C cases increased the hospital stays in our study.

\section{Conclusion}

Despite the observable variations in the clinical and laboratory findings among the COVID-19 pediatric patients admitted to the GPW and PICU, no serious consequences were observed. Patients with a reported history of SOB and low oxygen saturation at the ER had a higher chance of being admitted to the PICU.

\section{Acknowledgments}

The authors are thankful to the Institute of Research and Consulting Studies at King Khalid University, Saudi Arabia, for supporting this research financially through the grant \# 4-N-20/21. 


\section{Funding}

This research was funded by the Institute of Research and Consulting Studies at King Khalid University through grant number \# 4-N-20/21.

\section{Disclosure}

The authors declare no competing interests.

\section{References}

1. Wang C, Horby PW, Hayden FG, Gao GF. A novel coronavirus outbreak of global health concern. Lancet. 2020;395:470-473. doi:10.1016/S0140-6736(20)30185-9

2. Lu H, Stratton C, Tang Y. Outbreak of pneumonia of unknown etiology in Wuhan, China: the mystery and the miracle. $J$ Med Virol. 2020;92:401-402. doi:10.1002/jmv.25678

3. Dong L, Bouey J. Public mental health crisis during COVID-19 pandemic, China. Emerg Infect Dis. 2020;26:1616-1618. doi:10.3201/eid2607.200407

4. Li Q, Guan X, Wu P, et al. Early transmission dynamics in Wuhan, China, of Novel Coronavirus-infected pneumonia. $N$ Engl $J$ Med. 2020;382:1199-1207. doi:10.1056/NEJMoa2001316

5. Sung H, Roh KH, Hong KH, et al. COVID-19 molecular testing in Korea: practical essentials and answers from experts based on experiences of emergency use authorization assays. Ann Lab Med. 2020;40:439-447. doi:10.3343/alm.2020.40.6.439

6. Epidemiology Working Group for NCIP Epidemic Response, Chinese CDC. The epidemiological characteristics of an outbreak of 2019 novel coronavirus diseases (COVID-19) -China. Zhonghua Liu Xing Bing Xue Za Zhi. 2020;41:145-151. doi:10.3760/cma.j. issn.0254-6450.2020.02.003

7. Holshue ML, DeBolt C, Lindquist S, et al. First case of 2019 novel coronavirus in the United States. N Engl J Med. 2020;382:929-936. doi:10.1056/NEJMoa2001191

8. Rothe C, Schunk M, Sothmann P, et al. Transmission of 2019-nCoV infection from an asymptomatic contact in Germany. $N$ Engl $J$ Med 2020;382:970-971. doi:10.1056/NEJMc2001468

9. Torres-Navarro I, Abril-Pérez C, Roca-Ginés J, Sánchez-Arráez J, Botella- Estrada R, Évole-buselli M. Comment on two cases of COVID-19 presenting with a clinical picture resembling chilblains: first report from the Middle East': pernio unrelated to COVID-19. Clin Exp Dermatol. 2020;45:752-754. doi:10.1111/ced.14255

10. Giovanetti M, Benvenuto D, Angeletti S, Ciccozzi M. The first two cases of 2019-nCoV in Italy: where they come from? J Med Virol. 2020;92:518-521. doi:10.1002/jmv.25699

11. Ali AS, Al-Hakami AM, Shati AA, Asseri AA, Al-Qahatani SM. Salient conclusive remarks on epidemiology and clinical manifestations of pediatric COVID-19: narrative review. Front Pediatr. 2020;8:584694. doi:10.3389/fped.2020.584694

12. Liu W, Zhang Q, Chen J, et al. Detection of Covid-19 in children in early January 2020 in Wuhan, China. $N$ Engl J Med. 2020;382 (14):1370-1371. doi:10.1056/NEJMc2003717

13. Loconsole D, Caselli D, Centrone F, et al. Sars-cov-2 infection in children in southern Italy: a descriptive case series. Int $J$ Environ Res Public Health. 2020;17(17):1-7. doi:10.3390/ijerph17176080

14. Chao JY, Derespina KR, Herold BC, et al. Clinical characteristics and outcomes of hospitalized and critically ill children and adolescents with Coronavirus disease 2019 at a Tertiary Care Medical Center in New York City. J Pediatr. 2020;223:14-19.e2. doi:10.1016/j.jpeds.2020.05.006

15. Bixler D, Miller AD, Mattison CP, Taylor B, Komatsu K. SARSCoV-2-associated deaths among persons aged 21 years-United States, February 12-July 31, 2020. MMWR. 2020;69(37):1324 1329. doi: $10.15585 / \mathrm{mmwr} . \mathrm{mm} 6937 \mathrm{e} 4$
16. Dong Y, Dong Y, Mo X, et al. Epidemiology of COVID-19 among children in China. Pediatrics. 2020;145(6):e20200702. doi:10.1542/ peds.2020-0702

17. Patel NA. Pediatric COVID-19: systematic review of the literature. Am J Otolaryngol. 2020;41(5):102573. doi:10.1016/j. amjoto.2020.102573

18. Maltezou HC, Magaziotou I, Dedoukou X, et al. Children and adolescents with SARS-CoV-2 infection: epidemiology, clinical course and viral loads. Pediatr Infect Dis J. 2020;39(12):E388-92. doi:10.1097/INF.0000000000002899

19. Al-Hanawi MK, Angawi K, Alshareef N, et al. Knowledge, attitude and practice toward COVID-19 among the public in the Kingdom of Saudi Arabia: a cross-sectional study. Front Public Health. 2020;8:217. doi:10.3389/fpubh.2020.00217

20. Alsofayan YM, Althunayyan SM, Khan AA, Hakawi AM, Assiri AM. Clinical characteristics of COVID-19 in Saudi Arabia: a national retrospective study. $J$ Infect Public Health. 2020;13 (7):920-925. doi:10.1016/j.jiph.2020.05.026

21. Al-Tawfiq JA, Memish ZA. COVID-19 in the Eastern Mediterranean Region and Saudi Arabia: prevention and therapeutic strategies. Int $J$ Antimicrob Agents. 2020;55(5):105968. doi:10.1016/j. ijantimicag.2020.105968

22. Alrasheed H, Althnian A, Kurdi H, Al-Mgren H, Alharbi S. COVID19 spread in Saudi Arabia: modeling, simulation and analysis. Int $J$ Environ Res Public Health. 2020;17(21):7744. doi:10.3390/ ijerph17217744

23. Saudi Ministry of Health $(\mathrm{MOH})$. Hospital admission criteria for COVID-19 pediatric patients, version $1.1 ; 2020$. 1-3. Available from: https://www.moh.gov.sa/Ministry/MediaCenter/Publications/ Documents/Hospital-admission-criteria.pdf. Accessed May 8, 2021.

24. Saudi Center for Disease Control and Prevention. Quick interim guide to COVID-19 surveillance case definitions and disposition; 2020. Available from: https://covid19.cdc.gov.sa. Accessed May 8, 2021.

25. Saudi Ministry of Health. Protocol for adults patients suspected of/ confirmed with COVID-19 supportive care and antiviral treatment of suspected or confirmed COVID-19 infection Saudi MoH Protocol for Adults Patients Suspected of/Confirmed with COVID-19. Supportive Care. 2020;1-6.

26. Center for Disease Control and Prevention, Center for Preparedness and Response. Multisystem Inflammatory Syndrome in Children (MIS-C) associated with Coronavirus disease 2019 (COVID-19), Clinician Outreach and Communication (COCA) webinar; 2020. Available from: https://emergency.cdc.gov/coca/calls/2020/callinfo_051920.asp? deliveryName=USCDC_1052-DM28623. Accessed May 19, 2020.

27. Weiss SL, Peters MJ, Alhazzani W, et al. Surviving sepsis campaign international guidelines for the management of septic shock and sepsis-associated organ dysfunction in children. Intensive Care Med. 2020;46:10-67.

28. Bourkhissi L, Fakiri KEL, Nassih H, et al. Laboratory abnormalities in children with novel Coronavirus disease 2019. Clin Med Insights Pediatr. 2020;14:117955652095517. doi:10.1177/1179556520955177

29. Zhou F, Yu T, Du R, et al. Clinical course and risk factors for mortality of adult inpatients with COVID-19 in Wuhan, China: a retrospective cohort study. Lancet (London, England). 2020;395 (10229):1054-1062. doi:10.1016/S0140-6736(20)30566-3

30. Almutairi A, Al-shamrani A, Sultan P, Military M, Arabia S. Overview of COVID 19 in children with the current situation in Saudi Arabia. Medicina. 2020;80(6):704-714.

31. Alyami MH, Naser AY, Orabi MAA, Alwafi H, Alyami HS. Epidemiology of COVID-19 in the Kingdom of Saudi Arabia: an ecological study. Front Public Health. 2020;8(September):1-9.

32. Swann OV, Holden KA, Turtle L, et al. Clinical characteristics of children and young people admitted to hospital with covid-19 in United Kingdom: prospective multicentre observational cohort study. BMJ. 2020;370:m3249. doi:10.1136/bmj.m3249 
33. Li B, Zhang S, Zhang R, Chen X, Wang Y, Zhu C. Epidemiological and clinical characteristics of COVID-19 in children: a systematic review and meta-analysis. Front Pediatr. 2020;8(November):1-12. doi:10.3389/fped.2020.591132

34. Nathan N, Prevost B, Sileo C, et al. The wide spectrum of COVID-19 clinical presentation in children. J Clin Med. 2020;9(9):2950. doi: $10.3390 /$ jcm 9092950

35. King JA, Whitten TA, Bakal JA, McAlister FA. Symptoms associated with a positive result for a swab for SARS-CoV-2 infection among children in Alberta. Can Med Assoc J. 2021;193(1):E1-E9. doi:10.1503/cmaj.202065
36. Administration F and D. FDA cautions against use of hydroxychloroquine or chloroquine for COVID-19 outside of the hospital setting or a clinical trial due to risk of heart rhythm problems [Internet]; 2021. Available from: https://www.fda.gov/drugs/fda-drug-safety-pod casts/fda-cautions-against-use-hydroxychloroquine-or-chloroquinecovid-19-outside-hospital-setting-or. Accessed May 8, 2021.

37. Venturini E, Montagnani C, Garazzino S, et al. treatment of children with COVID-19: position paper of the Italian Society of Pediatric Infectious Disease. Ital J Pediatr. 2020;46(1):139. doi:10.1186/ s13052-020-00900-w

\section{Publish your work in this journal}

The International Journal of General Medicine is an international, peer-reviewed open-access journal that focuses on general and internal medicine, pathogenesis, epidemiology, diagnosis, monitoring and treatment protocols. The journal is characterized by the rapid reporting of reviews, original research and clinical studies across all disease areas. The manuscript management system is completely online and includes a very quick and fair peer-review system, which is all easy to use. Visit http://www.dovepress.com/ testimonials.php to read real quotes from published authors. 